\title{
A Characterization for Compact Sets in the Space of Fuzzy Star-Shaped Numbers with $L_{p}$ Metric
}

\author{
Zhitao Zhao' ${ }^{1}$ and Congxin $\mathrm{Wu}^{2}$ \\ ${ }^{1}$ School of Mathematical Sciences, Heilongjiang University, Harbin 150080, China \\ ${ }^{2}$ Department of Mathematics, Harbin Institute of Technology, Harbin 150001, China \\ Correspondence should be addressed to Zhitao Zhao; zhitaozhao0808@126.com
}

Received 15 January 2013; Accepted 12 April 2013

Academic Editor: Marco Donatelli

Copyright (C) 2013 Z. Zhao and C. Wu. This is an open access article distributed under the Creative Commons Attribution License, which permits unrestricted use, distribution, and reproduction in any medium, provided the original work is properly cited.

By means of some auxiliary lemmas, we obtain a characterization of compact subsets in the space of all fuzzy star-shaped numbers with $L_{p}$ metric for $1 \leq p<\infty$. The result further completes and develops the previous characterization of compact subsets given by Wu and Zhao in 2008 .

\section{Introduction}

Since the concept of fuzzy numbers was firstly introduced in 1970s, it has been studied extensively from many different aspects of the theory and applications such as fuzzy algebra, fuzzy analysis, fuzzy topology, fuzzy logic, and fuzzy decision making. Many applications restrict their description to fuzzy numbers, often implicitly, because of powerful fuzzy convexity of fuzzy numbers. This fuzzy convexity is mainly reflected on the convexity of the level sets of fuzzy numbers. However, apart from possible applications, it is of independent interest to see how far the supposition of convexity can be weakened without losing too much structure. Star-shapedness is a fairly natural extension to convexity. Surprisingly, many topological properties of spaces of compact star-shaped sets are similar to those of their compact convex counterparts. It is well known that star-shapedness also plays an important role in the theory and applications, such as nonsmooth analysis, approximation problems and optimization problems, (see [1-11]). Based on the importance of star-shapedness, as a corresponding extension to fuzzy numbers, fuzzy star-shaped numbers have been payed more and more attention, such as Chanussot et al. [12], Diamond [13], Diamond and Kloeden [14], Qiu et al. [15], and $\mathrm{Wu}$ and Zhao [16].

In this regard, Diamond has done a lot of work. In 1990, he firstly introduced the concept of fuzzy star-shaped numbers and defined metrics on the space of fuzzy starshaped numbers in [13]. Especially, he studied the properties of $L_{p}$ metric for $1 \leq p<\infty$. The induced metric spaces $\left(S_{0}^{n}, d_{p}\right)$ were shown to be separable, but not complete for $1 \leq p<\infty$. Finally, compact sets in $\left(S_{0}^{n}, d_{p}\right)$ were also characterized. Later on, Wu and Zhao [16] pointed out that the characterization of Diamond was incorrect by a counterexample and gave a correct characterization for compact sets in spaces $\left(S_{0}^{n}, d_{p}\right)$ for $1 \leq p<\infty$.

The aim of this paper is to further improve the characterization of compact sets in [16] and obtain a characterization of compact sets in the space of all fuzzy star-shaped numbers with $L_{p}$ metric for $1 \leq p<\infty$. The result of this paper will provide some help for future research on fuzzy star-shaped numbers.

\section{Preliminaries}

In $\mathbb{R}^{n}$, denote the Euclidean norm by $\|\cdot\|$, and denote the class of all nonempty compact sets in $\mathbb{R}^{n}$ by $\mathbb{K}^{n}$. If $A, B$ are nonempty compact sets in $\mathbb{R}^{n}$, then the Hausdorff distance between $A$ and $B$ is given by $d_{H}(A, B)=$ $\max \left\{\sup _{a \in A} \inf _{b \in B}\|a-b\|, \sup _{b \in B} \inf _{a \in A}\|a-b\|\right\}$. 
Definition 1 (see [14]). An element $K \in \mathbb{K}^{n}$ is star-shaped relative to a point $x \in K$ if for each $y \in K$, the line segment $\overline{x y}$ joining $x$ to $y$ is contained in $K$. The $\operatorname{ker} K$ of $K$ is the set of all points $x \in K$ such that the line segment $\overline{x y} \subset K$ for each $y \in K$ and co $K$ is the convex hull of $K$.

For a fuzzy set $u: \mathbb{R}^{n} \rightarrow[0,1]$, we suppose that

(1) $u$ is normal; that is, there exists an $x_{0} \in \mathbb{R}^{n}$ such that $u\left(x_{0}\right)=1$,

(2) $u$ is upper semicontinuous,

(3) supp $u=\operatorname{cl}\left\{x \in \mathbb{R}^{n}: u(x)>0\right\}$ is compact,

(4) $u$ is fuzzy star-shaped; that is, there exists $x \in \mathbb{R}^{n}$ such that $u$ is fuzzy star-shaped with respect to $x$, namely, for any $y \in \mathbb{R}^{n}$ and $0 \leq \lambda \leq 1$,

$$
u(\lambda y+(1-\lambda) x) \geq u(y)
$$

$\left(4^{\circ}\right) u$ is fuzzy star-shaped with respect to the origin.

Definition 2 (see [16]). A fuzzy star-shaped number is a fuzzy set $u: \mathbb{R}^{n} \rightarrow[0,1]$ satisfying (1), (2), (3), and (4). Let $S^{n}$ be the family of all fuzzy star-shaped numbers, and let $S_{0}^{n}$ be the family of all fuzzy sets which satisfy (1), (2), (3), and (4 ${ }^{\circ}$. Clearly, $S_{0}^{n} \subset S^{n}$.

For a fuzzy star-shaped number $u \in S^{n}$, we define its $\alpha$ level set as follows:

$$
[u]^{\alpha}= \begin{cases}\left\{x \in \mathbb{R}^{n}: u(x) \geq \alpha\right\}, & \text { if } 0<\alpha \leq 1, \\ \operatorname{supp} u, & \text { if } \alpha=0 .\end{cases}
$$

Letting $m \in \mathbb{R}^{n}$, we define

$$
\widehat{m}(x)= \begin{cases}1, & \text { if } x=m, \\ 0, & \text { if } x \neq m .\end{cases}
$$

It is obvious that $\widehat{m} \in S^{n}$ for each $m \in \mathbb{R}^{n}$.

We will define addition and scalar multiplication of fuzzy shaped-numbers levelsetwise; that is, for $u, v \in S^{n}$ and $c \geq 0$

$$
\begin{gathered}
{[u+v]^{\alpha}=[u]^{\alpha}+[v]^{\alpha},} \\
{[c u]^{\alpha}=c[u]^{\alpha}}
\end{gathered}
$$

for each $\alpha \in[0,1]$. It directly follows that $u+v, c u \in S^{n}$.

Now, let us recall some properties of fuzzy star-shaped numbers which will be used in this paper.

Proposition 3. If $u \in S^{n}$, then

(1) $[u]^{\beta} \subseteq[u]^{\alpha} \subseteq[u]^{0}, 0 \leq \alpha \leq \beta \leq 1$,

(2) $[u]^{\alpha}$ is a compact set of $\mathbb{R}^{n}$ for each $0 \leq \alpha \leq 1$,

(3) for any $\alpha \in(0,1]$, if $\left\{\alpha_{k}\right\}$ is an increasing sequence of real numbers in $(0,1]$ converging to $\alpha$, then $[u]^{\alpha}=$ $\bigcap_{k=1}^{\infty}[u]^{\alpha_{k}}$.

The following property derives directly from Definitions 1 and 2 .
Proposition 4 (see [14]). For any $u \in S^{n}, u$ is fuzzy starshaped with respect to $y$ if and only if $[u]^{\alpha}$ are star-shaped with respect to $y$ for all $\alpha \in[0,1]$.

Definition 5 (see [14]). Let $\operatorname{ker}(u)$ be the totality of $y \in \mathbb{R}^{n}$ such that $u$ is fuzzy star-shaped with respect to $y$. Define $f \operatorname{ker}(u)$ by $[f \operatorname{ker}(u)]^{\alpha}=\operatorname{ker}[u]^{\alpha}$ for a fuzzy star-shaped number $u$.

Proposition 6 (see [14]). For a fuzzy star-shaped number $u$, $\operatorname{ker}(u)$ is a convex set in $\mathbb{R}^{n}$, and $f \operatorname{ker}(u)$ is a fuzzy convex set which is normal; that is, $[f \operatorname{ker}(u)]^{1} \neq \emptyset$.

Definition 7 (see [14]). For each $1 \leq p<\infty$, one defines

$$
d_{p}(u, v)=\left(\int_{0}^{1} d_{H}\left([u]^{\alpha},[v]^{\alpha}\right)^{p} \mathrm{~d} \alpha\right)^{1 / p},
$$

for all $u, v \in S^{n}$, then $d_{p}$ is called the $L_{p}$ metric on $S^{n}$.

For the characterization of compact sets in $\left(S^{n}, d_{p}\right)$, the following definitions will be used in the sequel.

Definition 8 (see [14]). One says that $U \subset S^{n}$ is uniformly support bounded if the support sets $[u]^{0}$ are bounded in $\mathbb{R}^{n}$, uniformly for $u \in U$; that is, there is a constant $\mathscr{K}>0$ such that $d_{H}\left([u]^{0},\{\theta\}\right) \leq \mathscr{K}$ holds for all $u \in U$.

Definition 9 (see [14]). Let $u \in S^{n}$. If for any $\varepsilon>0$, there exists $\delta=\delta(u, \varepsilon)>0$ such that for all $0 \leq h<\delta$

$$
\int_{h}^{1} d_{H}\left([u]^{\alpha},[u]^{\alpha-h}\right)^{p} \mathrm{~d} \alpha<\varepsilon^{p}, \quad 1 \leq p<\infty,
$$

then one says that $u$ is $p$-mean left-continuous. If for nonempty $U \subset S^{n}$, the above inequality holds uniformly for $u \in U$, one says that $U$ is $p$-mean equi-left-continuous.

Definition 10 (see [17]). One says that $U \subset S^{n}$ is uniformly $p$-mean bounded if there is a constant $\mathscr{K}>0$ such that $d_{p}(u, \widehat{\theta}) \leq \mathscr{K}$ for all $u \in U$, where $\widehat{\theta}$ denotes $u \in S^{n}$ with $u(\theta)=1$, and $u(x)=0$ for any $x \neq \theta$.

Remark 11. Note that uniform $p$-mean boundedness is weaker than uniform support boundedness, namely, if $U \subset$ $\left(S^{n}, d_{p}\right)$ is uniformly support bounded, then $U$ is uniformly $p$-mean bounded; however, the converse implication does not hold.

\section{Main Results}

Before proving our main result, we demonstrate some auxiliary lemmas. 
Lemma 12. Let $u \in S^{n}$, then the following properties hold:

(1) $\operatorname{ker}(u) \subset[u]^{1}$,

(2) for any $m \in \operatorname{ker}(u), u-\widehat{m}$ exists, and for any $\alpha \in[0,1]$, one has

$$
\begin{aligned}
& {[u-\widehat{m}]^{\alpha}=[u]^{\alpha}-\{m\},} \\
& {[u+\widehat{m}]^{\alpha}=[u]^{\alpha}+\{m\},}
\end{aligned}
$$

(3) for any $m \in \operatorname{ker}(u), u-\widehat{m} \in S_{0}^{n}$,

(4) if $\left\{m_{k}\right\} \subset \mathbb{R}^{n}$ converges to $m_{0} \in \mathbb{R}^{n}$, then $\left\{\widehat{m}_{k}\right\}$ converges to $\widehat{m}_{0}$ in $\left(S^{n}, d_{p}\right)$.

Proof. (1) Let $m \in \operatorname{ker}(u)$. By Definition 5, we know that for all $x \in \mathbb{R}^{n}$

$$
u(\lambda x+(1-\lambda) m) \geq u(x), \quad 0 \leq \lambda \leq 1 .
$$

Especially, the above inequality holds for each $x \in[u]^{1}$ and $\lambda=0$; that is, $u(m) \geq u(x)=1$. Thus, we get $m \in[u]^{1}$. Since $m$ is an arbitrary element of $\operatorname{ker}(u)$, then we have that $\operatorname{ker}(u) \subset[u]^{1}$.

(2) According to the Zadeh extension principle, we have

$$
(u-\widehat{m})(z)=\sup _{z=x-y} \min \{u(x), \widehat{m}(y)\}
$$

and so, it is clear that $u-\widehat{m}$ exists for each $m \in \operatorname{ker}(u)$. In the following, we infer that $[u-\widehat{m}]^{\alpha}=[u]^{\alpha}-\{m\}$ for each $\alpha \in[0,1]$.

Let $\alpha \in[0,1]$. For any $z \in[u]^{\alpha}-\{m\}$, there exists $x_{0} \in[u]^{\alpha}$ such that $z=x_{0}-m$. Then

$$
\begin{aligned}
(u-\widehat{m})(z) & =\sup _{z=x-y} \min \{u(x), \widehat{m}(y)\} \\
& \geq \min \left\{u\left(x_{0}\right), \widehat{m}(m)\right\} \\
& =u\left(x_{0}\right) \geq \alpha,
\end{aligned}
$$

and so, $z \in[u-\widehat{m}]^{\alpha}$. On the other hand, for any $z \in[u-\widehat{m}]^{\alpha}$, by the Zadeh extension principle, we get

$$
(u-\widehat{m})(z)=\sup _{z=x-y} \min \{u(x), \widehat{m}(y)\}=\sup _{z=x-m} u(x) \geq \alpha .
$$

Then, by the definition of the supremum, there exists $x_{k} \in$ $[u]^{0}$ for each $k \in \mathbb{N}$ such that

$$
u\left(x_{k}\right) \geq \alpha-\frac{1}{k}, \quad z=x_{k}-m .
$$

From the compactness of $[u]^{0},\left\{x_{k}\right\}$ has a subsequence $\left\{x_{k_{j}}\right\}$ converging to $x_{0} \in[u]^{0}$. Since $u$ is upper semicontinuous, then we have

$$
u\left(x_{0}\right) \geq \varlimsup_{j \rightarrow \infty} u\left(x_{k_{j}}\right) \geq \alpha .
$$

That is, $x_{0} \in[u]^{\alpha}$; thus, $z \in[u]^{\alpha}-\{m\}$.
Applying the similar techniques, we obtain $[u+\widehat{m}]^{\alpha}=$ $[u]^{\alpha}+\{m\}$ for each $\alpha \in[0,1]$.

(3) By Proposition 4, to prove $u-\widehat{m} \in S_{0}^{n}$, it is enough to verify that $[u-\widehat{m}]^{\alpha}$ is star-shaped with respect to the origin for each $\alpha \in[0,1]$, where $m$ is an arbitrary element of $\operatorname{ker}(u)$.

For any $x \in[u-\widehat{m}]^{\alpha}, \alpha \in[0,1]$; by statement (2), we have that $x \in[u]^{\alpha}-\{m\}$. So, we obtain that $x+m \in[u]^{\alpha}$. Since $m$ is an arbitrary element of $\operatorname{ker}(u)$, then by Definition 5 and Proposition 4 , the line segment $\overline{m(x+m)}$ joining $m$ to $x+m$ is contained in $[u]^{\alpha}$; that is,

$$
\lambda(x+m)+(1-\lambda) m=\lambda x+m \in[u]^{\alpha}, \quad 0 \leq \lambda \leq 1 .
$$

Thus, $\lambda x \in[u]^{\alpha}-\{m\}$ for all $0 \leq \lambda \leq 1$. That is, the line segment $\overline{\theta x} \subset[u]^{\alpha}-\{m\}$.

Consequently, $[u-\widehat{m}]^{\alpha}$ is star-shaped with respect to the origin for each $\alpha \in[0,1]$.

(4) Since $\left\{m_{k}\right\} \subset \mathbb{R}^{n}$ converges to $m_{0} \in \mathbb{R}^{n}$, then for every $\varepsilon>0$, there exists an integer $k_{0}$ such that when $k \geq k_{0}$

$$
\left\|m_{k}-m_{0}\right\|<\varepsilon
$$

Thus, for the above $\varepsilon>0$, we have

$$
\begin{aligned}
d_{p}\left(\widehat{m}_{k}, \widehat{m}_{0}\right) & =\left(\int_{0}^{1} d_{H}\left(\left[\widehat{m}_{k}\right]^{\alpha},\left[\widehat{m}_{0}\right]^{\alpha}\right)^{p} \mathrm{~d} \alpha\right)^{1 / p} \\
& =\left(\int_{0}^{1} d_{H}\left(\left\{m_{k}\right\},\left\{m_{0}\right\}\right)^{p} \mathrm{~d} \alpha\right)^{1 / p} \\
& =\left(\int_{0}^{1}\left\|m_{k}-m_{0}\right\|^{p} \mathrm{~d} \alpha\right)^{1 / p} \\
& <\varepsilon,
\end{aligned}
$$

whenever $k \geq k_{0}$. Therefore, $\left\{\widehat{m}_{k}\right\}$ converges to $\widehat{m}_{0}$ in $\left(S^{n}, d_{p}\right)$.

Lemma 13. For any $u \in\left(S^{n}, d_{p}\right), 1 \leq p<\infty, u$ is $p$-mean left-continuous.

Proof. The technique is similar to the proof of Lemma 3.1 in [16].

For every $u \in S^{n}$ and $r \in[0,1]$, denote

$$
u^{(r)}(x)= \begin{cases}u(x), & \text { if } u(x) \geq r \\ 0, & \text { if } u(x)<r\end{cases}
$$

Then, we have the following.

Lemma 14 (see [16]). A closed set $U \subset\left(S_{0}^{n}, d_{p}\right), 1 \leq p<\infty$, is compact if and only if

(1) $U$ is uniformly p-mean bounded,

(2) $U$ is p-mean equi-left-continuous,

(3) let $\left\{r_{i}\right\}$ be a decreasing sequence in $(0,1]$ converging to zero. For $\left\{u_{k}\right\} \subset U$, if $\left\{u_{k}^{\left(r_{i}\right)} \mid k=1,2,3, \ldots\right\}$ converges to $u\left(r_{i}\right) \in S_{0}^{n}$ in $d_{p}$, then there exists a $u_{0} \in S_{0}^{n}$ such that

$$
\left[u_{0}^{\left(r_{i}\right)}\right]^{\alpha}=\left[u\left(r_{i}\right)\right]^{\alpha}, \quad r_{i}<\alpha \leq 1 .
$$


Lemma 15. For any $u \in S^{n}, m \in \mathbb{R}^{n}$, and $0<r<1$, one has

$$
\widehat{m}^{(r)}=\widehat{m}, \quad(u+\widehat{m})^{(r)}=u^{(r)}+\widehat{m} .
$$

Proof. Let $\alpha$ be an arbitrary element in $[0,1]$.

If $r \leq \alpha \leq 1$, then $\left[\widehat{m}^{(r)}\right]^{\alpha}=[\widehat{m}]^{\alpha}=\{m\}$. If $0<\alpha<r$, then $\left[\widehat{m}^{(r)}\right]^{\alpha}=[\widehat{m}]^{r}=\{m\}=[\widehat{m}]^{\alpha}$. Hence, for each $\alpha \in$ $(0,1],\left[\widehat{m}^{(r)}\right]^{\alpha}=[\widehat{m}]^{\alpha}$, and so, it follows that $\left[\widehat{m}^{(r)}\right]^{0}=[\widehat{m}]^{0}$. This implies that $\widehat{m}^{(r)}=\widehat{m}$.

Similarly, if $r \leq \alpha \leq 1$, then by Lemma 12(2), we have

$$
\begin{aligned}
{\left[(u+\widehat{m})^{(r)}\right]^{\alpha} } & =[u+\widehat{m}]^{\alpha}=[u]^{\alpha}+\{m\} \\
& =\left[u^{(r)}\right]^{\alpha}+\{m\}=\left[u^{(r)}+\widehat{m}\right]^{\alpha} .
\end{aligned}
$$

If $0<\alpha<r$, then by Lemma 12(2), we have

$$
\begin{aligned}
{\left[(u+\widehat{m})^{(r)}\right]^{\alpha} } & =[u+\widehat{m}]^{r}=[u]^{r}+\{m\} \\
& =\left[u^{(r)}\right]^{\alpha}+\{m\}=\left[u^{(r)}+\widehat{m}\right]^{\alpha}
\end{aligned}
$$

Hence, for each $\alpha \in(0,1],\left[(u+\widehat{m})^{(r)}\right]^{\alpha}=\left[u^{(r)}+\widehat{m}\right]^{\alpha}$, and so, it follows that $\left[(u+\widehat{m})^{(r)}\right]^{0}=\left[u^{(r)}+\widehat{m}\right]^{0}$. This implies that $(u+\widehat{m})^{(r)}=u^{(r)}+\widehat{m}$.

Now, we give the characterization of compact sets in $\left(S^{n}, d_{p}\right)$ for $1 \leq p<\infty$.

Theorem 16. A closed set $U \subset\left(S^{n}, d_{p}\right), 1 \leq p<\infty$ is compact if and only if

(i) $U$ is uniformly p-mean bounded,

(ii) $U$ is p-mean equi-left-continuous,

(iii) let $\left\{r_{i}\right\}$ be a decreasing sequence in $(0,1]$ converging to zero. For $\left\{u_{k}\right\} \subset U$, if $\left\{u_{k}^{\left(r_{i}\right)} \mid k=1,2,3, \ldots\right\}$ converges to $u\left(r_{i}\right) \in S^{n}$ in $d_{p}$, then there exists a $u_{0} \in S^{n}$ such that

$$
\left[u_{0}^{\left(r_{i}\right)}\right]^{\alpha}=\left[u\left(r_{i}\right)\right]^{\alpha}, \quad r_{i}<\alpha \leq 1
$$

Proof. Necessity: (1) Since $U$ is compact in $\left(S^{n}, d_{p}\right)$, it follows that $U$ is a bounded set in $\left(S^{n}, d_{p}\right)$. This implies that $U$ is uniformly $p$-mean bounded.

(2) Let $\varepsilon>0$, and let $u_{1}, u_{2}, \ldots, u_{m} \in S^{n}$ be a $(\varepsilon / 3)$ net of $U$, that is, for any $u \in U$, there exists an element $u_{i}(1 \leq i \leq m)$ satisfying $d_{p}\left(u, u_{i}\right)<\varepsilon / 3$. By Lemma 12, $u_{1}, u_{2}, \ldots, u_{m}$ are $p$-mean left-continuous, and so, there exists $\delta(\varepsilon)=\min _{1 \leq i \leq m} \delta\left(u_{i}, \varepsilon\right)>0$ such that

$$
\int_{h}^{1} d_{H}\left(\left[u_{i}\right]^{\alpha},\left[u_{i}\right]^{\alpha-h}\right)^{p} \mathrm{~d} \alpha<\left(\frac{\varepsilon}{3}\right)^{p}
$$

for $i=1,2, \ldots, m$ and $0 \leq h<\delta(\varepsilon)$.
Thus, for $u \in U$, we can obtain by triangle inequality

$$
\begin{aligned}
\left(\int_{h}^{1} d_{H}\left([u]^{\alpha},[u]^{\alpha-h}\right)^{p} \mathrm{~d} \alpha\right)^{1 / p} & \\
\leq & \left(\int_{h}^{1} d_{H}\left([u]^{\alpha},\left[u_{i}\right]^{\alpha}\right)^{p} \mathrm{~d} \alpha\right)^{1 / p} \\
& +\left(\int_{h}^{1} d_{H}\left(\left[u_{i}\right]^{\alpha},\left[u_{i}\right]^{\alpha-h}\right)^{p} \mathrm{~d} \alpha\right)^{1 / p} \\
& +\left(\int_{h}^{1} d_{H}\left(\left[u_{i}\right]^{\alpha-h},[u]^{\alpha-h}\right)^{p} \mathrm{~d} \alpha\right)^{1 / p} \\
\leq & d_{p}\left(u_{i}, u\right)+\left(\int_{h}^{1} d_{H}\left(\left[u_{i}\right]^{\alpha},\left[u_{i}\right]^{\alpha-h}\right)^{p} \mathrm{~d} \alpha\right)^{1 / p} \\
& +d_{p}\left(u_{i}, u\right) \\
< & \frac{\varepsilon}{3}+\frac{\varepsilon}{3}+\frac{\varepsilon}{3} \\
= & \varepsilon .
\end{aligned}
$$

Therefore, $U$ is $p$-mean equi-left-continuous.

(3) According to the definition of $u^{\left(r_{i}\right)},\left[u^{\left(r_{i}\right)}\right]^{\alpha}=[u]^{\alpha}$ for each $r_{i} \leq \alpha \leq 1$, and $\left[u^{\left(r_{i}\right)}\right]^{\alpha}=[u]^{r_{i}}$ for each $0 \leq \alpha<r_{i}$. Since $u \in S^{n}$, then from Proposition 4 , there exists $x \in \mathbb{R}^{n}$ such that $[u]^{\alpha}$ is star-shaped with respect to $x$ for each $0 \leq \alpha \leq 1$. So, $\left[u^{\left(r_{i}\right)}\right]^{\alpha}$ is star-shaped with respect to $x$ for each $0 \leq \alpha \leq 1$; thus, it is obvious by Proposition 4 that $u^{\left(r_{i}\right)} \in S^{n}$ for each $i \in \mathbb{N}$.

For $\left\{u_{k}\right\} \subset U$, we assume that $\left\{u_{k}^{\left(r_{i}\right)}\right\}_{k=1}^{\infty}$ converges to $u\left(r_{i}\right) \in S^{n}$ in $d_{p}$. Since $U$ is compact in $\left(S^{n}, d_{p}\right)$, then $\left\{u_{k}\right\}$ has a subsequence $\left\{u_{k_{j}}\right\}$ converging to $u_{0} \in S^{n}$ in $d_{p}$. By Minkowski's inequality, it follows that

$$
\begin{aligned}
\left(\int_{r_{i}}^{1} d_{H}\left(\left[u_{0}\right]^{\alpha},\left[u\left(r_{i}\right)\right]^{\alpha}\right)^{p} \mathrm{~d} \alpha\right)^{1 / p} & \left(\int_{r_{i}}^{1} d_{H}\left(\left[u_{0}\right]^{\alpha},\left[u_{k_{j}}\right]^{\alpha}\right)^{p} \mathrm{~d} \alpha\right)^{1 / p} \\
& +\left(\int_{r_{i}}^{1} d_{H}\left(\left[u_{k_{j}}\right]^{\alpha},\left[u\left(r_{i}\right)\right]^{\alpha}\right)^{p} \mathrm{~d} \alpha\right)^{1 / p} \\
= & \left(\int_{r_{i}}^{1} d_{H}\left(\left[u_{0}\right]^{\alpha},\left[u_{k_{j}}\right]^{\alpha}\right)^{p} \mathrm{~d} \alpha\right)^{1 / p} \\
& +\left(\int_{r_{i}}^{1} d_{H}\left(\left[u_{k_{j}}^{\left(r_{i}\right)}\right]^{\alpha},\left[u\left(r_{i}\right)\right]^{\alpha}\right)^{p} \mathrm{~d} \alpha\right)^{1 / p} .
\end{aligned}
$$

But since

$$
\begin{gathered}
\lim _{j \rightarrow \infty}\left(\int_{r_{i}}^{1} d_{H}\left(\left[u_{0}\right]^{\alpha},\left[u_{k_{j}}\right]^{\alpha}\right)^{p} \mathrm{~d} \alpha\right)^{1 / p}=0, \\
\lim _{j \rightarrow \infty}\left(\int_{r_{i}}^{1} d_{H}\left(\left[u_{k_{j}}^{\left(r_{i}\right)}\right]^{\alpha},\left[u\left(r_{i}\right)\right]^{\alpha}\right)^{p} \mathrm{~d} \alpha\right)^{1 / p}=0,
\end{gathered}
$$


then we get

$$
\left(\int_{r_{i}}^{1} d_{H}\left(\left[u_{0}\right]^{\alpha},\left[u\left(r_{i}\right)\right]^{\alpha}\right)^{p} \mathrm{~d} \alpha\right)^{1 / p}=0,
$$

and this implies that

$$
d_{H}\left(\left[u_{0}\right]^{\alpha},\left[u\left(r_{i}\right)\right]^{\alpha}\right)=0,
$$

a.e. in $\left(r_{i}, 1\right]$. Thus, we obtain that

$$
\left[u_{0}\right]^{\alpha}=\left[u\left(r_{i}\right)\right]^{\alpha},
$$

a.e. in $\left(r_{i}, 1\right]$; that is, $\left[u_{0}\right]^{\alpha}=\left[u\left(r_{i}\right)\right]^{\alpha}$ for each $\left(r_{i}, 1\right] \backslash \Omega$, where $\Omega \subset\left(r_{i}, 1\right]$ has Lebesgue measure zero and clearly $\left(r_{i}, 1\right] \backslash \Omega$ is dense in $\left(r_{i}, 1\right]$. Let $\bar{\alpha} \in \Omega$, then there exists an increasing sequence $\alpha_{k} \nearrow \bar{\alpha}$ with $\alpha_{k} \in\left(r_{i}, 1\right] \backslash \Omega$ and $\left[u_{0}\right]^{\alpha_{k}}=\left[u\left(r_{i}\right)\right]^{\alpha_{k}}$. So

$$
\begin{aligned}
d_{H}\left(\left[u_{0}\right]^{\bar{\alpha}},\left[u\left(r_{i}\right)\right]^{\bar{\alpha}}\right) \\
\leq d_{H}\left(\left[u_{0}\right]^{\bar{\alpha}},\left[u_{0}\right]^{\alpha_{k}}\right)+d_{H}\left(\left[u_{0}\right]^{\alpha_{k}},\left[u\left(r_{i}\right)\right]^{\alpha_{k}}\right) \\
\quad+d_{H}\left(\left[u\left(r_{i}\right)\right]^{\alpha_{k}},\left[u\left(r_{i}\right)\right]^{\bar{\alpha}}\right) \\
=d_{H}\left(\left[u_{0}\right]^{\bar{\alpha}},\left[u_{0}\right]^{\alpha_{k}}\right)+d_{H}\left(\left[u\left(r_{i}\right)\right]^{\alpha_{k}},\left[u\left(r_{i}\right)\right]^{\bar{\alpha}}\right) .
\end{aligned}
$$

By Proposition 3, both terms on the right converge to zero as $k \rightarrow \infty$, so $d_{H}\left(\left[u_{0}\right]^{\bar{\alpha}},\left[u\left(r_{i}\right)\right]^{\bar{\alpha}}\right)=0$, and hence $\left[u_{0}\right]^{\bar{\alpha}}=$ $\left[u\left(r_{i}\right)\right]^{\bar{\alpha}}$ for each $\bar{\alpha} \in \Omega$.

Consequently, we have $\left[u_{0}^{\left(r_{i}\right)}\right]^{\alpha}=\left[u_{0}\right]^{\alpha}=\left[u\left(r_{i}\right)\right]^{\alpha}$ for each $r_{i}<\alpha \leq 1$.

Sufficiency: From Lemma 12(3), we have that $\{u-\widehat{m}: u \in$ $U, m \in \operatorname{ker}(u)\} \subset S_{0}^{n}$. We divide the rest of the proof into two steps.

Step 1. We prove that $\{u-\widehat{m}: u \in U, m \in \operatorname{ker}(u)\}$ satisfies (1)-(3) in Lemma 14.

Firstly, we show that if $U$ is uniformly $p$-mean bounded, then $\{\operatorname{ker}(u): u \in U\}$ is uniformly bounded. Otherwise, we infer that for any $k>0, k \in \mathbb{N}$; we can find that $u_{k} \in U$ such that

$$
d_{H}\left(\operatorname{ker}\left(u_{k}\right),\{\theta\}\right)>k \text {. }
$$

By Proposition 3(1) and Lemma 12(1), it follows that

$$
\begin{gathered}
\left(\int_{0}^{1} d_{H}\left(\left[u_{k}\right]^{\alpha},\{\theta\}\right)^{p} \mathrm{~d} \alpha\right)^{1 / p} \\
\quad \geq\left(\int_{0}^{1} d_{H}\left(\left[u_{k}\right]^{1},\{\theta\}\right)^{p} \mathrm{~d} \alpha\right)^{1 / p} \\
\quad \geq\left(\int_{0}^{1} d_{H}\left(\operatorname{ker}\left(u_{k}\right),\{\theta\}\right)^{p} \mathrm{~d} \alpha\right)^{1 / p} \\
>\left(\int_{0}^{1} k^{p} \mathrm{~d} \alpha\right)^{1 / p}=k, \quad k \in \mathbb{N} .
\end{gathered}
$$

This contradicts condition (i).
Secondly, we conclude that $\{u-\widehat{m}: u \in U, m \in \operatorname{ker}(u)\}$ is uniformly $p$-mean bounded. According to condition (i) and the conclusion in last paragraph, we obtain that $\{\operatorname{ker}(u): u \in$ $U\}$ is uniformly bounded, and then there exists a $\mathscr{K}>0$ such that for all $u \in U$

$$
d_{H}(\operatorname{ker}(u),\{\theta\}) \leq \mathscr{K}
$$

By the condition (i), there exists a $\mathscr{K}^{\prime}>0$ such that for all $u \in U$

$$
\left(\int_{0}^{1} d_{H}\left([u]^{\alpha},\{\theta\}\right)^{p} \mathrm{~d} \alpha\right)^{1 / p} \leq \mathscr{K}^{\prime} .
$$

Thus, by Lemma 12(2) for all $u \in U$, we get

$$
\begin{aligned}
&\left(\int_{0}^{1} d_{H}\left([u-\widehat{m}]^{\alpha},\{\theta\}\right)^{p} \mathrm{~d} \alpha\right)^{1 / p} \\
&=\left(\int_{0}^{1} d_{H}\left([u]^{\alpha}-\{m\},\{\theta\}\right)^{p} \mathrm{~d} \alpha\right)^{1 / p} \\
&=\left(\int_{0}^{1} d_{H}\left([u]^{\alpha},\{m\}\right)^{p} \mathrm{~d} \alpha\right)^{1 / p} \\
& \leq\left(\int_{0}^{1} d_{H}\left([u]^{\alpha},\{\theta\}\right)^{p} \mathrm{~d} \alpha\right)^{1 / p} \\
&+\left(\int_{0}^{1} d_{H}(\{\theta\},\{m\})^{p} \mathrm{~d} \alpha\right)^{1 / p} \\
& \leq\left(\int_{0}^{1} d_{H}\left([u]^{\alpha},\{\theta\}\right)^{p} \mathrm{~d} \alpha\right)^{1 / p} \\
&+\left(\int_{0}^{1} d_{H}(\{\theta\}, \operatorname{ker}(u))^{p} \mathrm{~d} \alpha\right)^{1 / p} \\
& \leq \mathscr{K}^{\prime}+\mathscr{K} .
\end{aligned}
$$

This implies that condition (1) of Lemma 14 is satisfied.

Thirdly, we verify that $\{u-\widehat{m}: u \in U, m \in \operatorname{ker}(u)\}$ is $p$ mean equi-left-continuous. In fact, since $U$ is $p$-mean equileft-continuous, then for every $\varepsilon>0$, there exists a $\delta>0$ such that for all $0 \leq h \leq \delta$ and $u \in U$

$$
\int_{h}^{1} d_{H}\left([u]^{\alpha},[u]^{\alpha-h}\right)^{p} \mathrm{~d} \alpha<\varepsilon^{p},
$$

and so, we have

$$
\begin{aligned}
\int_{h}^{1} d_{H}\left([u-\widehat{m}]^{\alpha},[u-\widehat{m}]^{\alpha-h}\right)^{p} \mathrm{~d} \alpha \\
\quad=\int_{h}^{1} d_{H}\left([u]^{\alpha}-\{m\},[u]^{\alpha-h}-\{m\}\right)^{p} \mathrm{~d} \alpha \\
\quad=\int_{h}^{1} d_{H}\left([u]^{\alpha},[u]^{\alpha-h}\right)^{p} \mathrm{~d} \alpha<\varepsilon^{p} .
\end{aligned}
$$

Hence, condition (2) of Lemma 14 holds. 
Finally, we prove that condition (3) of Lemma 14 is also satisfied. Let $\left\{r_{i}\right\}$ be a decreasing sequence in $(0,1]$ converging to zero. We suppose that $\left\{\left(u_{k}-\widehat{m}_{k}\right)^{\left(r_{i}\right)}\right\} \quad\left(u_{k} \in U, m_{k} \in \operatorname{ker}\left(u_{k}\right)\right)$ converges to $u^{*}\left(r_{i}\right) \in S_{0}^{n}$ in $d_{p}$. Denote $v_{k}=u_{k}-\widehat{m}_{k}$. Then, $\left\{v_{k}^{\left(r_{i}\right)}\right\}$ converges to $u^{*}\left(r_{i}\right) \in S_{0}^{n}$ in $d_{p}$. By Lemma 15 , we have

$$
u_{k}^{\left(r_{i}\right)}=\left(v_{k}+\widehat{m}_{k}\right)^{\left(r_{i}\right)}=v_{k}^{\left(r_{i}\right)}+\widehat{m}_{k} \text {. }
$$

By the preceding proof, $\{\operatorname{ker}(u) \mid u \in U\}$ is uniformly bounded. Then, $\left\{m_{k}\right\}$ has a subsequence $\left\{m_{k_{j}}\right\}$ converging to $m_{0} \in \mathbb{R}^{n}$, and so by Lemma 12(4), $\left\{u_{k_{j}}^{\left(r_{i}\right)}\right\}=\left\{v_{k_{j}}^{\left(r_{i}\right)}+\widehat{m}_{k_{j}}\right\}$ converges to $u^{*}\left(r_{i}\right)+\widehat{m}_{0}$ in $\left(S^{n}, d_{p}\right)$. By condition (iii) and Lemma 12(2), there exists a $u_{0} \in S^{n}$ such that

$$
\left[u_{0}^{\left(r_{i}\right)}\right]^{\alpha}=\left[u^{*}\left(r_{i}\right)+\widehat{m}_{0}\right]^{\alpha}=\left[u^{*}\left(r_{i}\right)\right]^{\alpha}+\left\{m_{0}\right\},
$$

wherever $r_{i}<\alpha \leq 1$, and so by Lemma 12(2), we have

$$
\begin{aligned}
{\left[u^{*}\left(r_{i}\right)\right]^{\alpha} } & =\left[u_{0}^{\left(r_{i}\right)}\right]^{\alpha}-\left\{m_{0}\right\}=\left[u_{0}\right]^{\alpha}-\left\{m_{0}\right\} \\
& =\left[u_{0}-\widehat{m}_{0}\right]^{\alpha}=\left[\left(u_{0}-\widehat{m}_{0}\right)^{\left(r_{i}\right)}\right]^{\alpha},
\end{aligned}
$$

wherever $r_{i}<\alpha \leq 1$.

Define $u_{0}^{*}=u_{0}-\widehat{m}_{0}$. Then, by Lemma 12(3), it follows that $u_{0}^{*} \in S_{0}^{n}$. Thus, condition (3) of Lemma 14 holds.

Step 2. We infer that $U$ is a compact set in $\left(S^{n}, d_{p}\right)$.

Let $\left\{u_{k}\right\} \subset U$. Since $\{u-\widehat{m}: u \in U, m \in \operatorname{ker}(u)\}$ is a subset of $\left(S_{0}^{n}, d_{p}\right)$ and satisfies condition (1)-(3) of Lemma 14, then $\{u-\widehat{m}: u \in U, m \in \operatorname{ker}(u)\}$ is a compact set of $\left(S_{0}^{n}, d_{p}\right)$, and so for fixed $m_{k} \in \operatorname{ker}\left(u_{k}\right)(k \in \mathbb{N}),\left\{u_{k}-\widehat{m}_{k}\right\}$ has a subsequence $\left\{u_{k_{j}}-\widehat{m}_{k_{j}}\right\}$ converging to $u_{0} \in\left(S_{0}^{n}, d_{p}\right)$. By the proceeding proof, $\{\operatorname{ker}(u): u \in U\}$ is uniformly bounded, so $\left\{m_{k_{j}}\right\}$ has a subsequence $\left\{m_{k_{j(l)}}\right\}$ converging to $m_{0} \in \mathbb{R}^{n}$. It is obvious that $\left\{u_{k_{j(l)}}-\widehat{m}_{k_{j(l)}}\right\}$ converges to $u_{0} \in\left(S_{0}^{n}, d_{p}\right)$.

Denote $v_{k_{j(l)}}=u_{k_{j(l)}}-\widehat{m}_{k_{j(l)}}$. Then, $\left\{v_{k_{j(l)}}\right\}$ converges to $u_{0} \in\left(S_{0}^{n}, d_{p}\right)$. Therefore, by Lemma 12(4), we get that $\left\{u_{k_{j(l)}}\right\}=$ $\left\{v_{k_{j(l)}}+\widehat{m}_{k_{j(l)}}\right\}$ converges to $u_{0}+\widehat{m}_{0} \in\left(S^{n}, d_{p}\right)$, which completes the proof.

\section{Conclusion}

Since star-shapedness has played an important role in the theory and applications of classical analysis, such as nonsmooth analysis, approximation problems, and optimization problems, then as an extension to fuzzy numbers, fuzzy star-shaped numbers should be also payed more and more attention. In this paper, we further complete and develop the previous result in [16] and give a characterization of compact subsets in the space of all fuzzy star-shaped numbers with $L_{p}$ metric for $1 \leq p<\infty$. The result of this paper will provide some help for future research on the theory of fuzzy starshaped numbers.

\section{Acknowledgments}

The authors would like to thank the anonymous reviewers for their helpful comments which improved the original paper. The project is supported by the Youth Scientific Funds of Heilongjiang University (no. QL201007) and NSFC (nos. 11201128 and 11226255).

\section{References}

[1] J. Cel, "An optimal Krasnosel'skii-type theorem for an open starshaped set," Geometriae Dedicata, vol. 66, no. 3, pp. 293-301, 1997.

[2] J. Cel, "Representations of starshaped sets in normed linear spaces," Journal of Functional Analysis, vol. 174, no. 2, pp. 264$273,2000$.

[3] F. H. Clarke, Optimization and Nonsmooth Analysis, John Wiley \& Sons, New York, NY, USA, 1983.

[4] I. Ekeland, "Nonconvex minimization problems," Bulletin of the American Mathematical Society, vol. 1, no. 3, pp. 443-474, 1979.

[5] P. M. Gruber and T. I. Zamfirescu, "Generic properties of compact starshaped sets," Proceedings of the American Mathematical Society, vol. 108, no. 1, pp. 207-214, 1990.

[6] D. A. Klain, "Invariant valuations on star-shaped sets," Advances in Mathematics, vol. 125, no. 1, pp. 95-113, 1997.

[7] A. Kosinski, "Note on star-shaped sets," Proceedings of the American Mathematical Society, vol. 13, no. 6, pp. 931-933, 1962.

[8] F. Maccheroni, "Homothetic preferences on star-shaped sets," Decisions in Economics and Finance, vol. 24, no. 1, pp. 41-47, 2001.

[9] H. Mohebi and E. Naraghirad, "Cone-separation and starshaped separability with applications," Nonlinear Analysis: Theory, Methods \& Applications, vol. 69, no. 8, pp. 2412-2421, 2008.

[10] S. Park and J. Yoon, "Remarks on fixed point theorems on starshaped sets," Journal of the Korean Mathematical Society, vol. 18, no. 2, pp. 135-140, 1982.

[11] A. Shveidel, "Separability of star-shaped sets and its application to an optimization problem," Optimization, vol. 40, no. 3, pp. 207-227, 1997.

[12] J. Chanussot, I. Nyström, and N. Sladoje, "Shape signatures of fuzzy star-shaped sets based on distance from the centroid," Pattern Recognition Letters, vol. 26, no. 6, pp. 735-746, 2005.

[13] P. Diamond, "A note on fuzzy starshaped fuzzy sets," Fuzzy Sets and Systems, vol. 37, no. 2, pp. 193-199, 1990.

[14] P. Diamond and P. Kloeden, Metric Spaces of Fuzzy Sets, World Scientific, River Edge, NJ, USA, 1994.

[15] D. Qiu, L. Shu, and Z.-W. Mo, “On starshaped fuzzy sets," Fuzzy Sets and Systems, vol. 160, no. 11, pp. 1563-1577, 2009.

[16] C. Wu and Z. Zhao, "Some notes on the characterization of compact sets of fuzzy sets with $L_{p}$ metric," Fuzzy Sets and Systems, vol. 159, no. 16, pp. 2104-2115, 2008.

[17] M. Ma, "Some notes on the characterization of compact sets in $\left(E^{n}, d_{p}\right)$," Fuzzy Sets and Systems, vol. 56, no. 3, pp. 297-301, 1993. 


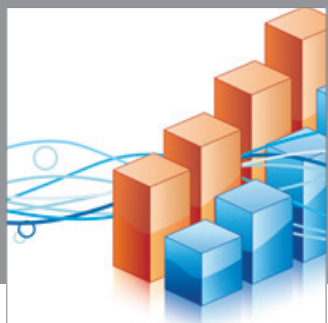

Advances in

Operations Research

mansans

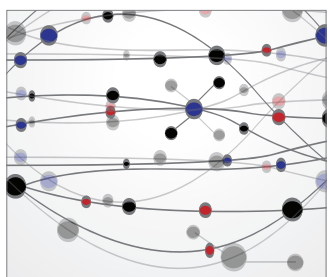

The Scientific World Journal
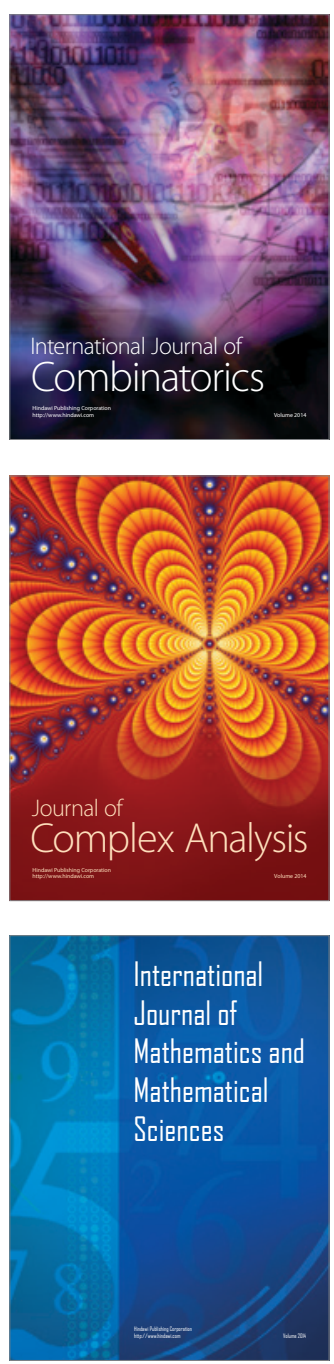
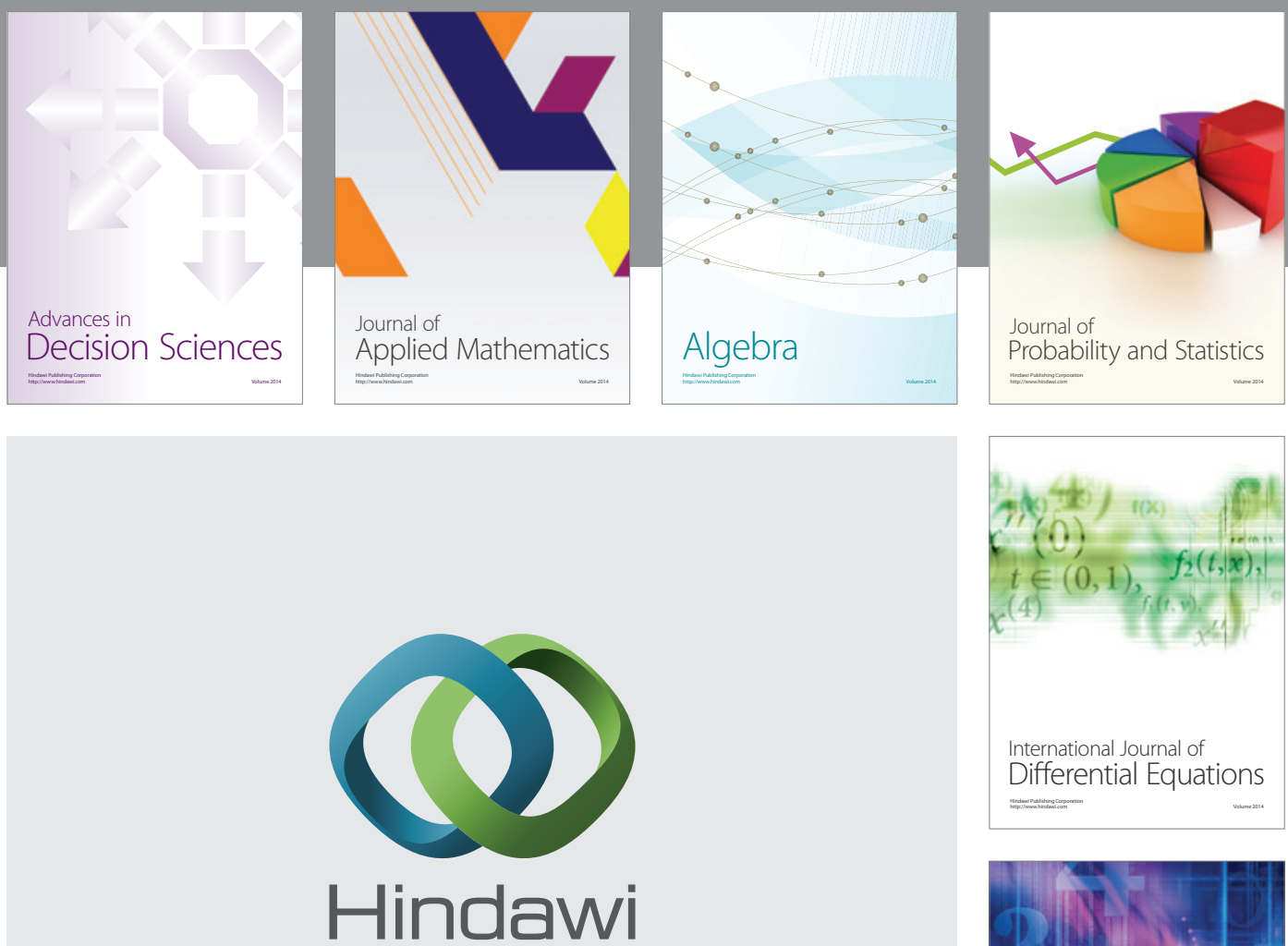

Submit your manuscripts at http://www.hindawi.com
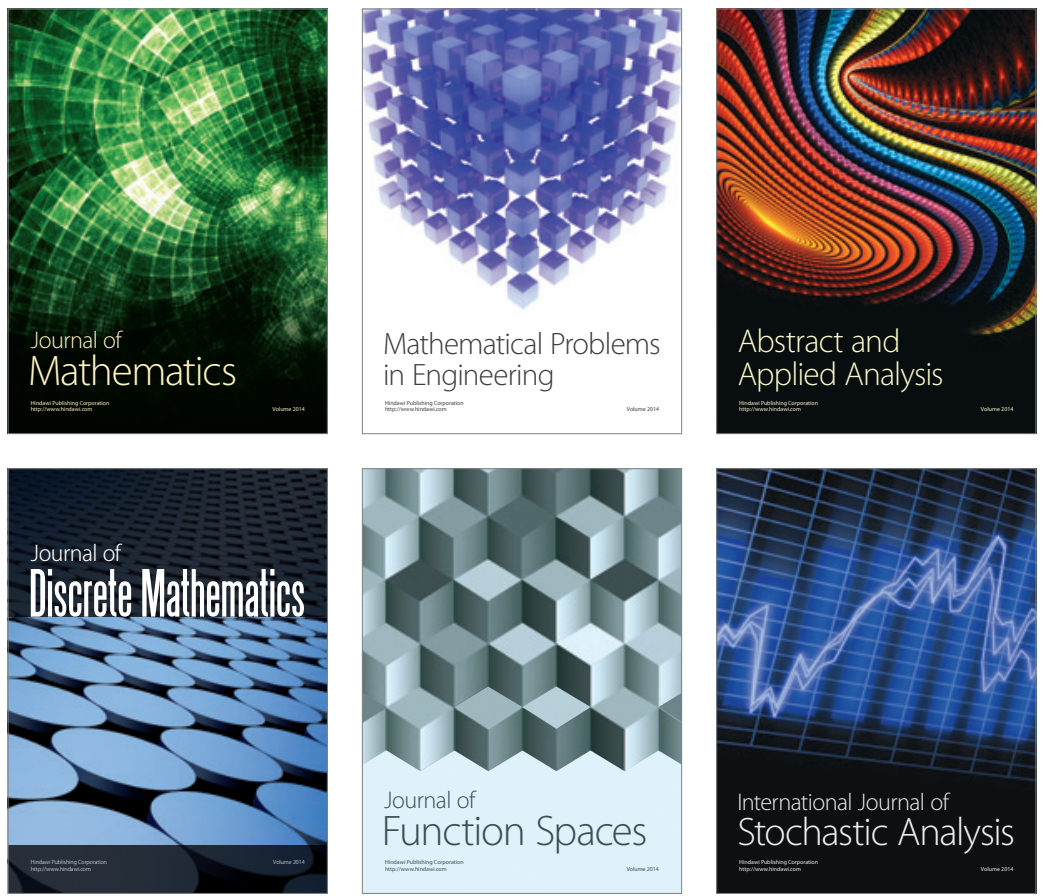

Journal of

Function Spaces

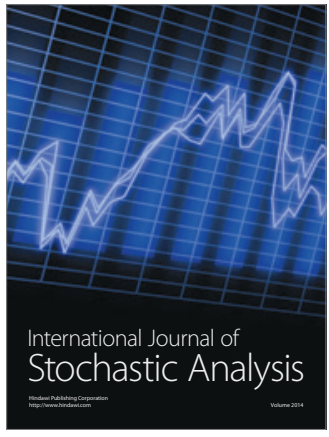

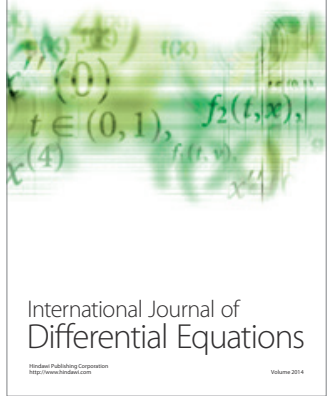
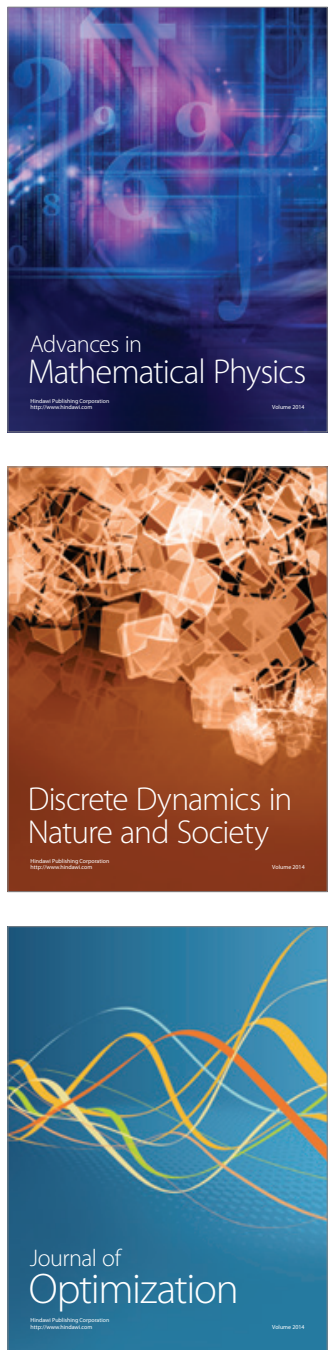